Original

\title{
La Iniciativa Open Access (OAI) en la literatura científica
}

\author{
Francisco M. Sánchez-Martín, Félix Millán Rodríguez, Humberto Villavicencio Mavrich
}

Servicio de Urología. Fundació Puiguert. Barcelona. España

\begin{abstract}
Resumen
Según la declaración de Budapest sobre la iniciativa Open Access (OAI) se define como un modelo editorial en el que el acceso a la literatura científica y su uso son gratuitos. Internet permite la libre circulación de información y ha sido la base de esta iniciativa. Las declaraciones de Bethesda y de Berlín, secundadas por diversos organismos investigadores internacionales proponen exigir a los investigadores que depositen una copia de todos sus artículos publicados en un autoarchivo o en un repositorio Open Access; y alentar a los investigadores a publicar sus artículos de investigación en revistas que permitan libre acceso a sus contenidos. En el presente trabajo se revisan las claves de la OAI, con sus aspectos positivos y sus controversias; y se analiza la posición de las bases de datos, buscadores y repositorios de información biomédica, así como la actitud de científicos, empresas editoriales y publicaciones periódicas. Hasta la actualidad la revista Actas Urológicas Españolas ofrece sus contenidos en Open Access en su versión On Line en español e inglés.
\end{abstract}

Palabras clave: OAI, Iniciativa Open Access. Literatura Científica. Repositorio. Publicación Electrónica.

\section{The Open Access Initiative (OAI) in the scientific literature}

\section{Abstract}

According to the declaration of the Budapest Open Access Initiative (OAI) is defined as a editorial model in which access to scientific journal literature and his use are free. Free flow of information allowed by Internet has been the basis of this initiative. The Bethesda and the Berlin declarations, supported by some international agencies, proposes to require researchers to deposit copies of all articles published in a self-archive or an Open Access repository, and encourage researchers to publish their research papers in journals Open Access. This paper reviews the keys of the OAI, with their strengths and controversial aspects; and it discusses the position of databases, search engines and repositories of biomedical information, as well as the attitude of the scientists, publishers and journals. So far the journal Actas Urológicas Españolas (Act Urol Esp) offer their contents on Open Access as On Line in Spanish and English.

Keywords: OAI. Open Access Initiative. Scientific Literature. Repository. Electronic Papers.

$\mathrm{E}$ 1 informe resultante de la Cumbre Mundial sobre la Sociedad de la Información, auspiciada por la ONU que tuvo lugar en Ginebra en 2003, reconoce que "la ciencia desempeña un papel cardinal en el desarrollo de la Sociedad de la Información. Gran parte de los elementos constitutivos de esta sociedad son el fruto de los avances científicos y técnicos que han sido posibles gracias a la comunicación mutua de los resultados de la investigación”. Más adelante, hace "un llamamiento a la solidaridad digital, en los planos nacional e internacional" 1 . Esta visión señala las coordenadas generales en las que se sitúa la información científica en la Era Digital: La ciencia como bien común, y el acceso a los resultados científicos como un derecho.

Internet ha revolucionado el panorama de la comunicación en el siglo XXI, con una dimensión comparable a la que tuvo la imprenta como medio de transmisión del conocimiento a toda la humanidad en el siglo XV. Los recursos bibliográficos electrónicos asociados a Internet han permitido a la comunidad científica hacer públicos y compartir los datos de sus investigaciones sin tener que cederlos incondicionalmente a las editoriales.

Estos conceptos han servido de preámbulo para el nacimiento de una nueva manera de entender la comunicación científica: La Iniciativa Open Access (OAI) (Fig. 1).

La OAI se define como un modelo en el que el acceso a la literatura científica es gratuito y de acceso permanente ${ }^{2}$. Internet es el soporte que ha permitido el intercambio libre de información científica y que ha puesto en crisis el concepto tradicional de la propiedad intelectual en el sector. La iniciativa 


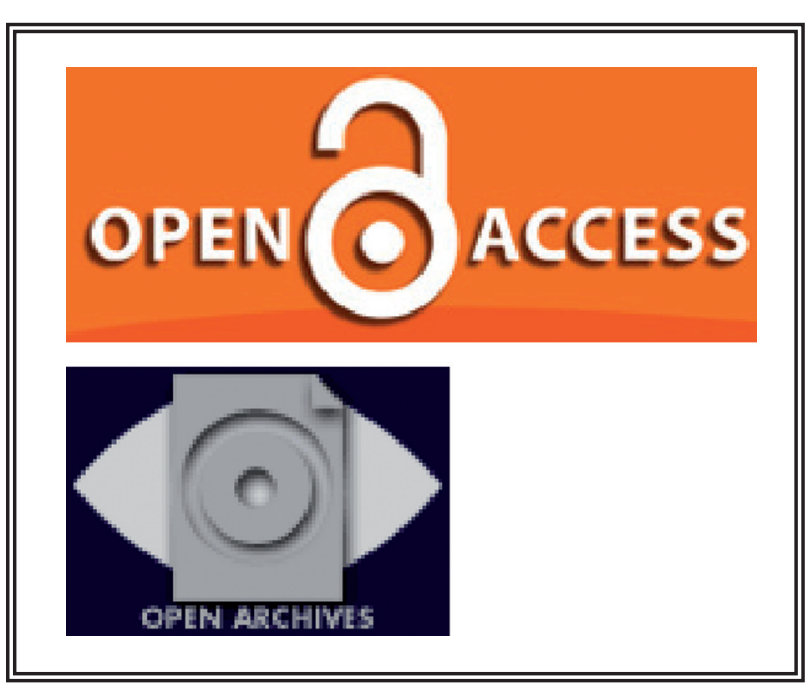

FIGURA 1. Iconos de las publicaciones adscritas al OAI y de los archivos Open Access.

también señala que las herramientas de software necesarias deben ser libremente accesibles y compatibles $^{3}$. Todo ello implica la libre circulación electrónica de contenidos científicos. Como señala Paola Gargiulo en la revista biomédica Il Pensiero: "Chi legge non paga" (Quien lee no paga) ${ }^{4}$. La Public Library of Science (PloS) define un modelo de Open Access basado en los siguientes principios: Uso ajustado a la licencia de acceso abierto, excelencia, integridad científica, impacto, cooperación, justicia financiera, participación comunitaria, internacionalismo y concepto de ciencia como recurso público ${ }^{5}$.

\section{Declaraciones}

De acuerdo con la Declaración de Budapest sobre OAI (BOAI), de diciembre del 2001, se entiende como Open Acces:

"La disponibilidad gratuita de la información científica en la Internet pública, para que cualquier usuario la pueda leer, descargar, copiar, distribuir, imprimir, con la posibilidad de buscar o enlazar todos los textos de los artículos, recorrerlos para indexación exhaustiva, usarlos como datos para software, o utilizarlos para cualquier otro propósito legal, sin barreras financieras, legales o técnicas, distintas de las fundamentales de la conexión a Internet" 6 .

Por su parte la Declaración de Berlín sobre Open Access (Open Access to Knowledge in the Sciences and Humanities), de octubre del 2003, la OAI se encuadra dentro del concepto más amplio posible en cuanto a fuentes de difusión del conocimiento humano y del patrimonio cultural que emana de la comunidad científica ${ }^{7}$. Fue promovida por la Max Planck Society y otras organizaciones europeas que financian proyectos de investigación. La declaración señala dos condiciones fundamentales para que un documento sea considerado Open Access:

1. Los autores y depositarios de la propiedad intelectual de tales contribuciones deben garantizar, a todos los usuarios por igual, el derecho gratuito, irrevocable y mundial de acceder a un trabajo erudito, lo mismo que licencia para copiarlo, usarlo, distribuirlo, transmitirlo y exhibirlo públicamente, y para hacer y distribuir trabajos derivativos, en cualquier medio digital para cualquier propósito responsable, todo sujeto al reconocimiento apropiado de autoría (los estándares de la comunidad continuarán proveyendo los mecanismos para hacer cumplir el reconocimiento apropiado y uso responsable de las obras publicadas), y el derecho a efectuar copias impresas en pequeño número para uso personal.

2. Debe depositarse una versión completa del trabajo y todos sus materiales complementarios, que incluya una copia del permiso del que se habla arriba, en un formato electrónico estándar, en, por lo menos, un repositorio online que utilice estándares técnicos para facilitar el libre acceso mantenido por una institución académica, sociedad erudita, agencia gubernamental, o una organización prestigiosa que busque promocionar el acceso abierto, distribución libre, intercambio y capacidad archivística a largo plazo de los originales.

La carta anima a las instituciones a desarrollar políticas para:

- Exigir a sus investigadores que depositen una copia de todos sus artículos publicados en un repositorio de acceso abierto.

- Alentar a sus investigadores a publicar sus artículos de investigación en revistas de acceso abierto.

La declaración señala asimismo una serie de actitudes ante el fenómeno Open Access:

- Estimular a los investigadores/beneficiarios de becas a publicar sus trabajos de acuerdo con los principios del paradigma Open Access.

- Estimular a los depositarios del patrimonio cultural para que apoyen el Open Access distribuyendo sus recursos a través de la Internet.

- Desarrollar medios y maneras de evaluar las contribuciones de Open Access y las revistas electrónicas, para mantener los estándares de garantía de calidad y práctica científica sana. 
- Abogar porque la publicación en Open Access sea reconocida como factor de evaluación para efectos de carrera profesional.

- Reclamar el mérito intrínseco de las contribuciones a una infraestructura de Open Access mediante el desarrollo de herramientas de software, provisión de contenido, creación de metadatos, o la publicación de artículos individuales.

La Declaración de Bethesda (Bethesda Statement on Open Access Publishing) de junio de 2003, promueve la IOA y recomienda a sus investigadores este tipo de planteo para publicar sus originales ${ }^{8}$. Entre una serie de consideraciones, propone unos consejos a los estamentos implicados. A las bibliotecas recomienda: Desarrollar mecanismos de apoyo a las publicaciones en acceso abierto, priorizar la docencia como objetivo del sistema Open Access, y facilitar catálogos de revistas de Open Access. A los editores recomienda un compromiso para proporcionar la opción Open Access entre los autores, así como establecer un calendario para la transición a modelos de Open Access, cooperar entre editores para desarrollar instrumentos que faciliten la publicación, almacenamiento y búsqueda de manuscritos en formato electrónico Open Access, y desarrollar estrategias que faciliten la adhesión a la OAI para instituciones en desventaja o países en desarrollo.

\section{Motivaciones y controversias}

El monopolio que las grandes editoriales han ejercido sobre la literatura científica durante buena parte del siglo XIX y la totalidad del XX ha ido derivando en diversos problemas: Las suscripciones a las revistas científicas son cada día más $\operatorname{caras}^{9}$, los autores sienten cada vez sus derechos menos respetados, los organismos financiadores de la investigación pierden la propiedad intelectual una vez deciden publicar los datos de sus observaciones, sin obtener otra cosa a cambio que el prestigio. Por su parte las empresas editoriales han obtenido beneficio económico a partir de las aportaciones de los autores y de los organismos a los que pertenecen aquéllos, y se han erigido en propietarias intelectuales de los contenidos.

El negocio montado alrededor de los artículos científicos es uno de los factores decisivos para la emergencia del OAI aunque el aspecto económico no parece ser el único motivo. Para Andrew Odlyzko, en su trabajo "Economic costs of toll access", los 10 billones de dólares anuales que ganan las editoriales, aún siendo una cifra enorme, sólo equivalen al $1 \%$ del gasto anual en investigación, que es de 1.000 billones de dólares, lo cual hace que no se contemple la necesidad de cambios radicales en muchos organismos investigadores ${ }^{10}$.

En el ámbito de las revistas de economía, el 60\% de todas las citas provienen de revistas cuyo coste representa a la biblioteca sólo el 10\% de su presupuesto en suscripciones ${ }^{11}$, es decir que la mayor parte del gasto se destina a revistas que no acaban influyendo a la comunidad científica. Muchos organismos no pueden seguir pagando y cancelan la suscripción a revistas en las que publican sus propios investigadores. Se produce entonces una paradoja: Los científicos y las organizaciones que sostienen la investigación, que en muchos casos proviene de fondos públicos, no pueden acceder a sus propios trabajos. Michael Eisen, uno de los promotores de la PLoS, se adhirió a la OAI después de que intentara montar una biblioteca On Line con datos sobre genómica y su correlación con clínica humana. Topó con la resistencia de los editores que le recordaron que vulneraba las leyes de propiedad intelectual. Como él miles de científicos, organizaciones y gobiernos se han adherido a la $\mathrm{OAI}^{12,13}$. Como ejemplo en España es posible citar el catálogo de revistas Open Acces del CSIC (e-Revistas.csic.org) ${ }^{14}$ o la iniciativa de la Comunidad de Madrid, que convierte en requerimiento obligatorio el volcado en un resositorio institucional abierto de los resultados de la investigación que vaya a financiar ${ }^{15}$.

Para Stevan Harnad, de Eprints.org con la OAI, la sociedad en su conjunto se beneficia de un ciclo de investigación más acelerado y la ciencia avanza de forma más eficaz porque los investigadores tienen acceso inmediato a los resultados de otros gru$\operatorname{pos}^{16}$ con el consiguiente aumento de la visibilidad y el intercambio inmediato de información. En 2001 Lawrence Lessing, directivo de PLoS y fundador de Creative Commons, observó que los artículos sobre computación accesibles en Open Acess tenían mucho más impacto que los que no usaban esta estrategia $^{17}$, lo que es útil para que universidades y autores atraigan inversiones.

La primera organización en poner a disposición libre contenidos científicos fue arXiv.org, promovida desde 1991 por Los Alamos National Laboratory (a veces llamada $x x x$, debido a que su servidor era 
xxx.lanl.gov), volcada desde 2001 a la biblioteca de la Cornell University. Entre las pioneras de la iniciativa figuran las Bibliotecas de la Universidad de Lund, que coordina y promueve el Directory of Open Access Journal (DOAJ), que contiene 1.278 revistas y una serie de organismos que figuran en la Tabla 1.

Pero no todo son ventajas, la Federation of American Societies for Experimental Biology (FASEB) y la propia Comisión Europea señalan que la calidad de las publicaciones pueden verse afectadas por un modelo que permita una apertura indiscriminada a la publicación de datos científicos sin garantía de tutela $^{12}$. Si esto se concreta puede frenarse el número de aportaciones que los científicos y las instituciones ceden en Open Acces. Ningún grupo de investigación consolidado puede renunciar a publicar sus datos en soportes sin garantías de calidad. Frente a esto algunos autores sólo entienden la iniciativa Open Access si está sujeta al peer-review ${ }^{18}$. Lawrence Lessing señala que la calidad de un repositorio Open Access está asegurada si se adoptan políticas de publicación consistentes ${ }^{19}$. Para el rector Gregory O'Brien, de la Association of American Universities (AAU), si no existe limitación alguna para reproducir los datos puede incrementarse la piratería, en detrimento de la producción original $^{20}$.
Las dificultades para valorar el impacto de los trabajos que se publican en Open Access hace necesario el desarrollo de nuevos marcadores bibliométricos ya que el Factor de Impacto (IF) presenta deficiencias como indicador ${ }^{21}$. Por otro lado, la volatilidad de la información aumentará si no se toman medidas que garanticen que un artículo almacenado en un autoarchivo o un repositorio no es modificado de forma sustancial a capricho de su autor.

No es lo mismo "gratuidad" que Open Access. La cuestión no está limitada a la reducción de la factura por suscripción. La OAI implica la gratuidad de acceso a los documentos, pero el proceso de captación, almacenaje, ordenación, mantenimiento búsqueda y dispensación de originales devenga unos costes que "alguien" debe asumir. Es previsible que el modelo de financiación futura cambie de forma sustancial: No se pagará por suscripción ni por acceso a los originales sino por publicación. El coste recaerá sobre los autores (o las instituciones que los contratan). Esto implica cambiar la mentalidad actual. La declaración de Bethesda entiende la publicación de los resultados de una investigación como una parte sustancial del proceso investigador, con su partida presupuestaria correspondiente. The FASEB Journal, revista que opera bajo suscripción, ofrece una opción Open Access a los autores (la financiación de ciertas investigaciones

Tabla 1. Organismos pioneros del apoyo a la OAI.

\begin{tabular}{llll}
\hline Organismo & País & Actividad & Papel \\
\hline arXiv.org & EEUU & Biblioteca & Inspirador \\
Universidad de Lund & Suecia & Biblioteca & Fundador \\
Max Plank Gesellschaft & Alemania & Investigación & Pionero \\
United Nations & Internacional & Política & Pionero \\
Centre National Recherche Scientifique (CNRS) & Francia & Investigación & Pionero \\
Institut National Santé et Recherche Médicale (INSERM) & Francia & Investigación & Pionero \\
Welcome Trust & Gran Bretaña & Investigación & Pionero \\
European Organization for Nuclear Research (CERN) & Suiza & Investigación & Pionero \\
Chinese Academy of Sciences & China & Investigación & Pionero \\
National Librery of Medicine (NLM)-PubMed & EEUU & Biblioteca & Apoyo \\
SciDevNet (Stanford University) & EEUU & Agencia Noticias & Adhesión \\
BioMed & EEUU & Directorio revistas OA & Adhesión \\
OAIster & EEUU & Directorio e-recursos & Adhesión \\
Creative Commons & EEUU & Derechos autor & Adhesión \\
eScholarship Repository & EEUU & Repositorio OA & Adhesión \\
Eprints sofware (Massachusetts University) & EEUU & Repositorio OA & Adhesión \\
Project Sherpa & Gran Bretaña & Biblioteca OA & Adhesión \\
\hline
\end{tabular}


va condicionada a la publicación Open Access), bajo tasa de 2.500 dólares por artículo ${ }^{22}$.

Los conceptos Open Access y Free Access asimismo son distintos. Mientras que aquél permite el libre acceso y el uso de las publicaciones, ésta permite el acceso pero acota el uso, limitándolo a las leyes de propiedad intelectual tradicionales.

La comunidad científica está evolucionando en masa hacia el modelo Open Access y se espera que alcance el $100 \%^{23}$. Se detecta, no obstante, una persistencia en el modelo tradicional conocida como "parálisis de Zeno", derivada de la necesidad que los autores tienen de publicar sus datos en revistas de prestigio reconocido y del miedo a la piratería y a la pérdida de credibilidad si lo hacen en soporte independiente ${ }^{24}$. El avance hacia un modelo abierto de comunicación científica no es una cuestión tecnológica sino social ${ }^{25}$.

Al margen de la presión ejercida por las grandes empresas editoriales, como amenaza directa a la OAI está el proyecto de ley H.R. 801, presentado por John Conyers en el congreso estadounidense, para limitar el derecho a la publicación en Internet de las organizaciones financiadas con fondos públicos. Esta propuesta ha movilizado a buen parte de la comunidad científica en defensa del OAI (Fig. 2).

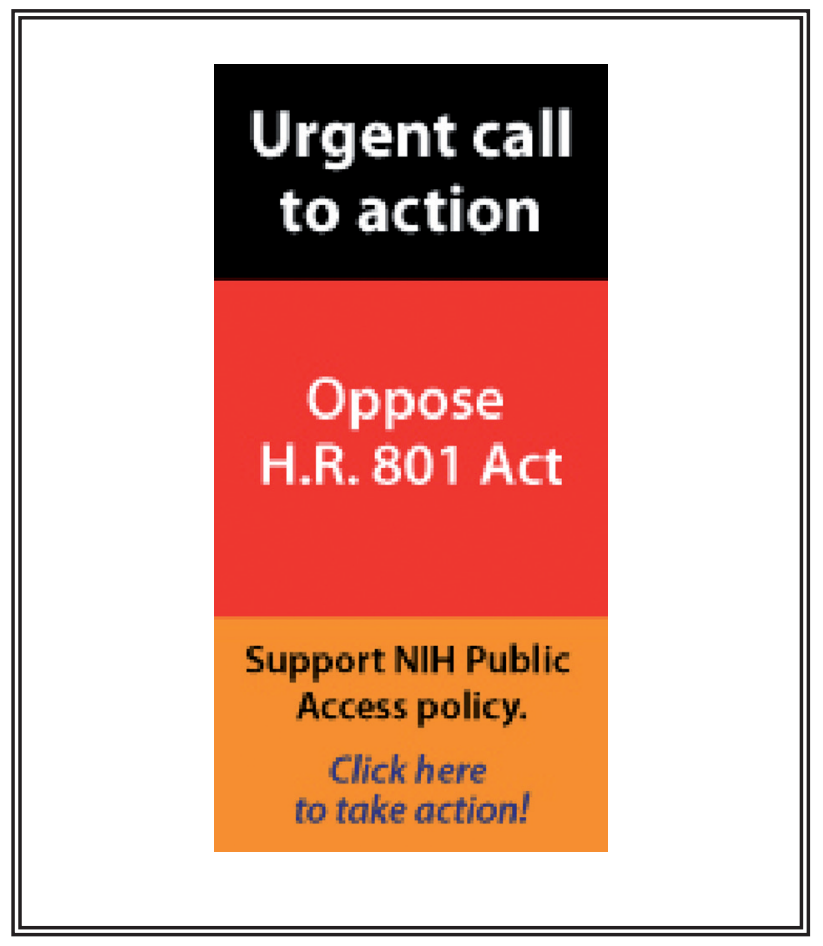

FIGURA 2. Iniciativa de la comunidad cientifica contra el proyecto de ley que limita la OAI.

\section{Repositorios y Autoarchivos}

Existen 2 fórmulas para publicar en Open Access $^{27}$ :

1. "Fórmula oro": Envío del trabajo a una revista que opere en Open Access, para que sea ella quien asuma el proceso editorial y la publicación.

2. "Fórmula verde": Archivo libre del trabajo en un sitio Web particular del autor o la institución (o comunidad de instituciones, por ejemplo la biblioteca).

Se adivina una tercera fórmula mixta: $\mathrm{El}$ original es publicado en una revista Open Access y queda archivado para libre disposición e indexado en los buscadores, tipo PubMed o ISI-Web of Knowledge (Wok) y al mismo tiempo es almacenado en un repositorio.

Para el sostenimiento de la OAI es imprescindible que la información se halle almacenada y ordenada en repositorios. Un repositorio es un sitio centralizado en un servidor donde se almacena y mantiene información digital, habitualmente bases de datos o archivos informáticos ${ }^{26}$. Puede entenderse como sinónimo de depósito, archivo o colección, los repositores facilitan la carga, ordenación, localización y descarga de contenidos. Suelen pertenecer a instituciones comprometidas con el OAI, por lo general bibliotecas, departamentos universitarios, organismos culturales, sociedades científicas, aunque cualquier autor puede desarrollar su propio repositorio.

Las instituciones investigadoras y algunos autores han desarrollado repositorios de almacenamiento de sus originales (eprints), dando lugar a un fenómeno llamado autoarchivo (self-archive) ${ }^{28}$. Es importante señalar que un autoarchivo no puede considerarse una publicación ${ }^{29}$. Para no colisionar con los intereses de las revistas, se adopta la fórmula de cargar como autoarchivo una versión previa a la publicación por ejemplo antes del peerreview (modalidades distintas de pre-print).

Los repositorios obligan a su sostenimiento económico y a su gestión. Para paliar estos inconvenientes han ido apareciendo repositorios generales. El más importante es el Directorio de Revistas Open Access (Directory of Open Access Journals, DOAJ), fundado por el Open Society Institute - Budapest (anfitrión de la Budapest Open Access Iniciativa). Reside en las bibliotecas de la Lund University (Suecia) ${ }^{30}$ financiado por la coalición SPARC (The Scholarly Publishing and Academic Resources Coalition). Los criterios de selección de del DOAJ figuran en Tabla 2. 
Tabla 2. Criterios de Selección de revistas Open Access del DOAJ

\section{Cobertura}

Temática: Todas las disciplinas científicas y académicas.

Tipos de recurso: Revistas científicas y académicas que publiquen trabajos de investigación o artículos de revisión a texto completo.

Fuentes: Procedentes de: organizaciones académicas, gubernamentales, comerciales, organismos sin ánimo de lucro.

Público objetivo: Principal sector a quien va dirigido, los investigadores.

Contenido: La mayoría de los artículos corresponderán a artículos de investigación. Todos los artículos serán accesibles a texto completo.

Acceso

Coste: El acceso será gratuito tanto para los usuarios como para las instituciones

Subscripción: Gratuita On Line.

Las revistas deben de estar exentas del periodo de embargo.

Es obligatorio que en los metadatos aparezca el título de la revista.

\section{Calidad}

Control de calidad: Los artículos de la revista contarán con un control de calidad a través de un editor, comité editorial o revisión por pares

\section{Periodicidad}

En cuanto al parámetro de periodicidad de las revistas, si bien no está mencionado dentro de los criterios de selección, el DOAJ, define el termino de "periodical" como una publicación seriada que publique a intervalos regulares, con carácter indefinido, en los que cada ejemplar se numere y date de forma consecutiva y que éstos números aparezcan al menos con una frecuencia de dos veces al año.

Existen otros repositorios como: eprints, cogPrints, PloS, BioMed, OAISter, Scholarship Repository, Dspace; y su número es creciente. En España existen diversas iniciativas de repositorios, la mayor parte dependientes de universidades ${ }^{26}$. La web E-revistas del Centro de Información y Documentación científica (CiNDOC), dependiente del CSIC, actúa como repositorio de sus contenidos generados y de catálogo de recursos Open Access ${ }^{31}$. Algunas editoriales se van adhiriendo de una $u$ otra forma a un modelo afin al OAI desarrollando sistemas de almacenaje de documentos, también garantizando el volcado de referencias en los buscadores y el volcado de contenidos en las grandes bases de datos biomédicas.

\section{Posición de PubMed e ISI-Wok}

La filosofía de gratuidad y libertad de acceso del buscador PubMed y sus listas de fondos coincide con la de la OAI. PubMed indica claramente al usuario aquellos contenidos que son accesibles de forma completa y gratuita (Full free text). Cuando una revista está adherida a la OAI aparece un link que señala esta condición, remitiendo a la revista (Free article at journal site) o remitiendo a PMC, la zona de contenidos gratuitos de PubMed (Free article in PMC) que contiene más de 450 revistas con un millón y medio de artículos de acceso libre ${ }^{32}$. Opcionalmente aparece un vínculo al directorio de revistas Open Access llamado BioMed Central (las revistas pagan por figurar en este directorio).
PubMed permite estrategias de búsqueda avanzada (Limits) para obtener únicamente los contenidos Open Access. PMC actúa asimismo como repositorio, tanto de artículos de revistas como de libros, aplicando una fórmula Free Access (acceso libre, aunque el uso no sea en todos los casos libre, si no restringido a los criterios de publicación de cada editorial). Advierte que “...los usuarios de PMC son responsables directos y únicos del cumplimiento de restricciones de derechos ..."33, que varían según se trate de originales publicados en revistas que acatan la OAI o en revistas que mantienen el clásico copyright.

Por su parte el buscador ISI-Wok es un sitio de acceso bajo cuota y no distingue si sus referencias corresponden a la OAI ni dispone de ningún catálogo de contenidos de Free Access.

\section{Propiedad intelectual}

En la OAI la propiedad existe pero el uso es libre. Sólo se señalan dos únicas limitaciones para el usuario: Reconocimiento del origen (citar a los autores), lo cual se conoce como derecho a la atribución (Atribution License); y limitar el número de copias para distribución. La BOAI incluye la recomendación de que el autor conserve el control sobre la integridad de su trabajo. Creative Commons, es la organización referente en la propiedad intelectual en $\mathrm{OAI}^{34}$. Una serie de iconos señalan las garantías a autores y usuarios (Figura 3). 


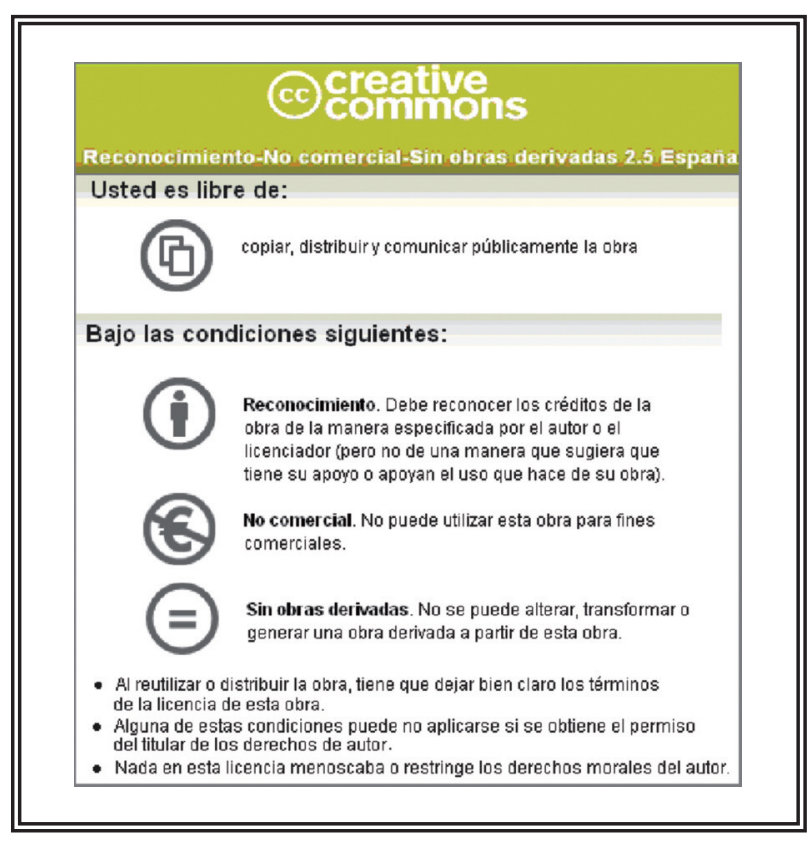

FIGURA 3. Condiciones de uso de los contenidos de Actas Urológicas Españolas On Line.

La liberalización en el uso de las publicaciones es asumida por el 70\% de las revistas clásicas. No aceptan la OAI de forma explícita pero permiten a los autores colgar sus artículos en repositorios una vez publicados (post-prints) ${ }^{29}$.

\section{Posición editorial}

Las grandes editoriales y muchas revistas científicas han percibido la importancia del fenómeno Open Access y se ha adherido a él de forma inmediata. En el catálogo de PMC figuran las siguientes instituciones con mayor o menor implicación en la cesión abierta de sus contenidos: BM Journal Un Lockheed, Elsevier Sponsor Hed Documenta, Yuca Open Access, Oxford Open, Portland Prez Opt2Pay, Springer Open Choice, Taylor \& Francis iOpen Access, y Wiley-Blackwell Online Open ${ }^{35}$.

No hay fórmula fija para la implicación Open Access de las revistas clásicas y cada una decide unilateralmente sus licencias. Algunas ofrecen sólo el último número en Open Access (Inmediate) mientras que otras liberan contendidos a 6, 12, 24 o 36 meses. Las licencias pueden ser cambiantes en el tiempo, en función de las tendencias del mercado. Otra fórmula es ofrecer en abierto sólo parte de los contenidos de un número. Las revistas urológicas internacionales de gran impacto se acogen a alguna de las formulas parciales de Open Access, dejando la mayor parte de sus referencias bajo pago (pay-per-view).
Como explica Antonio Lafuente, en su artículo divulgativo "Bien común y Open Access, "algunos equipos de redacción o grupos de autores han fundado su propia revista ante la imposibilidad de seguir trabajando en Open Access y se han registrado movimientos importantes en este sentido ${ }^{36}$. En diciembre de 2003 el consejo editorial del Journal of Algorithms (Elsevier) tras dimitir en pleno, difunde una Declaración de Independencia y anuncia el lanzamiento de ACM Transactions on Algorithms. El alto precio, según se explica en la carta de dimisión, fue también la causa de que los editores de Machine Learning Journal abandonaran Kluwer para pasar a convertirse en el Journal of Machine Learning Research European Economic Review (Elsevier) se transformó en el Journal of European Economic Association, Labor History (Taylor \& Francis) es ahora Labor, y Molecules (Springer Verlag) se sigue llamando Molecules pero ahora está con MDPI. En todos los casos, los editores redactan una declaración de independencia y solicitan a los autores que confiaban en la versión comercial de la revista que envien sus trabajos para ser difundidos en la modalidad Open Access" Otras corporaciones siguen directamente una política Open Access, como Hindawi Publishing Corporation ${ }^{37}$, con más de un centenar de revistas entre las que figura Advances in Urology. Por el contario, revistas que inicialmente se adhirieron a la iniciativa, como el British Medical Journal han regresado a una fórmula de acceso restringido parcial.

\section{Posición de Actas Urológicas Españolas}

La revista Actas Urológicas Españolas está en la actualidad adherida a la OAI y está indexada en el DOAJ, por lo tanto todos sus contendios desde septiembre de 1999 hasta ahora son de libre acceso y libre utilización, con derecho a atribución y limitación de copias para uso personal. Los artículos pueden ser consultados accediendo a la web de la revista (http://www. actasurologicas.info), utilizando el registro por fechas o el buscador por palabras clave. La revista está indexada en los principales buscadores, a través de los cuales se puede acceder a sus contenidos libremente en PubMed el vínculo a la revista se produce clicando sobre la fórmula "Free article at journal site" o sobre el icono FREE FULL TEXT in english/spanish (Figura 4) que señalan el acceso libre y gratuito a cada una de las referencias. 


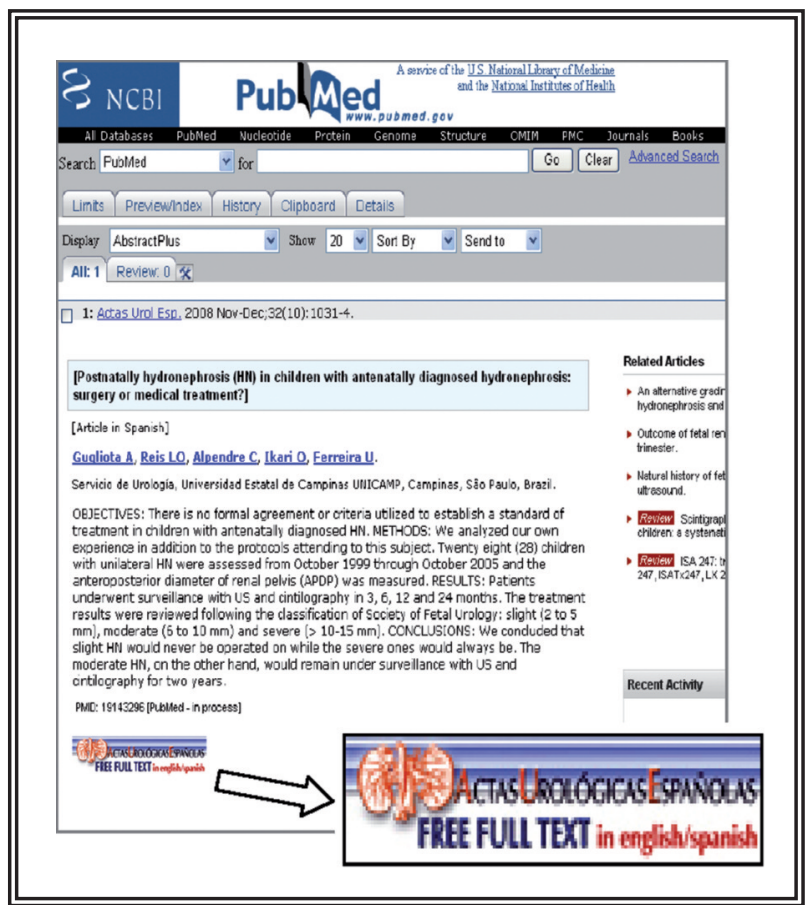

FIGURA 4. Icono-link Free Full Text de PubMed para un artículo de Actas Españolas de Urología.

Otros buscadores, como Scielo (http://scielo. isciii.es/scielo.php/lng_es) y Google Scholar (http:// scholar.google.es), localizan los contenidos de Actas mediante un link [PDF] pero sin iconos referentes a la OAI y Actas está incluida en el catálogo del DOAJ, indexada en el Journal Citation Report y en ISI-Wok pero sin iconos referentes a la OAI.

La fórmula de propiedad intelectual en estos momentos se adapta a la normativa de Creative Commons con la fórmula: Libre uso, reconocimiento como único requisito ${ }^{38}$. Mantiene su difusión en papel, acompañada de una versión electrónica completa (Actas Urológicas On Line) instalada en la Web, que actúa no sólo como directorio sino también como repositorio. También dispone de una pasarela electrónica para la recepción y gestión de originales.

Actas Españolas de Urología es una de las pocas publicaciones biomédicas españolas con una versión bilingüe. La totalidad de los contenidos publicados en español (en papel y formato electrónico) son traducidos al inglés (formato electrónico). Este servicio es gratuito para los autores. La revista mantiene actualmente una política de gratuidad para la aceptación de originales.

Ventajas como la adhesión al OAI, versión On Line, traducción al inglés y localización-indexación
Tabla 3. Ventajas de Actas Españolas de urología para los autores

\begin{tabular}{ll}
\hline Ventajas & Utilidad \\
\hline Pasarela electrónica & $\begin{array}{l}\text { Recepción de originales por } \\
\text { Internet }\end{array}$ \\
Filtro previo peer-review & $\begin{array}{l}\text { Favorecer la calidad de } \\
\text { contenidos }\end{array}$ \\
$\begin{array}{l}\text { Indexación en Bases } \\
\text { Datos On Line } \\
\text { (PubMed, ISI-Wok....) }\end{array}$ & $\begin{array}{l}\text { Favorecer visibilidad e } \\
\text { impacto }\end{array}$ \\
$\begin{array}{l}\text { Indexación en repositorios } \\
\text { (DOAJ) }\end{array}$ & $\begin{array}{l}\text { Favorecer acceso, uso y } \\
\text { visibilidad }\end{array}$ \\
$\begin{array}{l}\text { Publicación bilingüe } \\
\text { español-inglés }\end{array}$ & $\begin{array}{l}\text { Favorecer visibilidad e } \\
\text { impacto internacional }\end{array}$ \\
$\begin{array}{l}\text { Edición dual papel y } \\
\text { electrónica }\end{array}$ & $\begin{array}{l}\text { Favorecer visibilidad e } \\
\text { impacto }\end{array}$ \\
$\begin{array}{l}\text { Publicación en Open } \\
\text { Access }\end{array}$ & $\begin{array}{l}\text { Favorecer acceso, uso y } \\
\text { visibilidad a lectores. }\end{array}$ \\
\hline
\end{tabular}

en los principales buscadores internacionales permiten ofrecer a los autores más visibilidad e impacto para sus trabajos. Los lectores disponen de una plataforma de acceso y uso libre de los contenidos. En la Tabla 3 se resumen las ventajas de Actas Urológicas Españolas actualmente, como miembro de la OAI.

\section{CONCLUSIONES}

La OAI para acceso y utilización de información biomédica libre es un fenómeno cada vez más extendido. Internet es la base formal que sostiene esta iniciativa, impulsada por una serie de factores entre los que destacan el deseo de los autores y de las instituciones de liberarse del monopolio de las grandes empresas editoriales y la defensa de la libre circulación de la información científica como bien común. Los criterios generales de la OAI se hallan en las declaraciones de Budapest, Bethesda y Berlín, apoyados por la comunidad investigadora general y por organismos oficiales, universidades y centros de investigación. Las editoriales se han ido adhiriendo de forma paulatina a esta tendencia pero mantienen la propiedad intelectual de las publicaciones. Los autores y las instituciones que financian la investigación son partidarios de situar sus escritos en revistas Open Access, liberalizando los derechos de autor, especialmente cuando los datos se han obte- 
nido con financiación pública. Los repositorios (almacén) y autoarchivos actúan como plataformas electrónicas, para almacenar y facilitar el acceso y el uso público de las publicaciones. Una buena fórmula es que las editoriales se adhieran a la OAI y funcionen como repositorios. Algunos inconvenientes de la OAI son la posibilidad de deterioro de la calidad de los contenidos, el aumento de la piratería, las dificultades de sostenimiento del alojamiento.

\section{REFERENCIAS}

1. http://www.itu.int/wsis/outcome/booklet/declaration_Aes.html.

2. Parada A. El acceso abierto (open access) y el futuro de la edición en el ámbito biomédico: una figura con múltiples aristas. El profesional de la información 2005; 14: 326-334.

3. Melero M. Open access y repositorios institucionales: Nuevos sistemas y herramientas para la comunicación científica. CSIC. 2006. http://digital.csic.es/bitstream/10261/3035/1/Cursorepositorios-melero.pdf.

4. http://www.pensiero.it/attualita/articolo.asp?ID_sezione $=31 \&$ ID_articolo $=773$.

5. http://www.plos.org/about/principles.html.

6. http://www.soros.org/openaccess/read.shtml.

7. http://oa.mpg.de/openaccess-berlin/berlindeclaration.html.

8. http://www.earlham.edu/ peters/fos/bethesda.htm\#summary.

9. http://www.lessig.org/blog/2009/03/john_conyers_and_ open_access.html.

10. www.dtc.umn.edu/ odlyzko/doc/OpenAccess_book-odlyzkochapter.pdf.

11. Willinsky, J. The access principle: the case for open access to research and scholarship. Cambridge, MA; London: MIT Press, 2005. xix, 285 pp. ISBN 0-262-23242-1 £22.95. http://informationr.net/ir/reviews/revs202.html.

12. El cambio de paradigma de la e-ciencia. COMISIÓN DE LAS COMUNIDADES EUROPEAS Bruselas, 5.3.2009, Pag 3. http: / / eur-lex.europa.eu/LexUriServ/LexUriServ.do?uri= COM:2009:0108:FIN:ES:PDF.

13. http://www.earlham.edu/ peters/fos/2009/03/mit-adoptsuniversity-wide-oa-mandate.html.

14. http://www.erevistas.csic.es/buscador_externo.php

15. ORDEN 679/2009, de 19 de febrero, por la que se establecen las bases reguladoras de ayudas a programas de actividades de I + D entre grupos de investigación de la Comunidad de Madrid y convocatoria en tecnologías cofinanciada con Fondo Social Europeo. B.O.C.M. Núm. 53 4-5-09, pag. 13.

16. http://www.eprints.org/.
17. Lawrence, S, (2001) Online or Invisible?, Nature 411 (2001) (6837): 521. http://www.neci.nec.com/lawrence/papers/online-nature01/.

18. http://www.earlham.edu/ peters/fos/fosblog.html

19. http://www.americanscientist.org/bookshelf/pub/lawrencelessig.

20. Gregory O'Brien, Chancellor. University of New Orleans. Testimony before the Subcommittee on Telecommunications, Trade, and Consumer Protection. U.S. House of Representatives Committee on Commerce. H.R. 1858. Consumer and Investor Access to Information Act of 1999. June 15, 1999. http://www. aau.edu/.

21. Hitchcock, S. (2007) "Effect of open access on citation impact: a bibliography of studies." http://opcit.eprints.org/oacitationbiblio.html. Hitchcock, S. (2007) "Effect of open access on citation impact: a bibliography of studies." Available http://opcit. eprints.org/oacitation-biblio.html.

22. http://www.fasebj.org/misc/edpolicies.shtml\#Open_ Access_Option_for_Authors_.

23. Harnad, S. (2006) Opening Access by Overcoming Zeno's Paralysis. In: Open Access: Key Strategic, Technical and Economic Aspects, Chandos. http://users.ecs.soton.ac.uk/harnad/ Temp/harnad-jacobsbook.html. http://eprints.ecs.soton.ac. uk/12094/.

24. http://openaccess.eprints.org/index.php?/archives/44Open-Access-and-Zenos-Paralysis.html

25. http://www.nature.com/nature/focus/accessdebate/7.html

26. http://www2.ub.edu/bid/consulta_articulos.php?fichero=20meler4.htm.

27. http://www.eprints.org/openaccess/.

28. http://www.dtc.umn.edu/ odlyzko/.

29. http://www.eprints.org/openaccess/self-faq/\#self-archivingvs-publication.

30. http://www.lub.lu.se/en/about-lub.html.

31. http://www.erevistas.csic.es/buscador_externo.php.

32. http://www.pubmedcentral.nih.gov/.

33. http://www.pubmedcentral.nih.gov/about/authorms.html.

34. http://creativecommons.org/.

35. http://www.pubmedcentral.nih.gov/about/openftlist.html.

36. Lafuente A. Bien común y Open Access. http://www.madrimasd.org/informacionIdi/analisis/analisis/analisis.asp?id=15 $498 \&$ sec $=17$.

37. http://www.hindawi.com/.

38. http://creativecommons.org/licenses/by-nc-nd/2.5/es/.

Correspondencia autor: Dr. Francisco M. Sánchez-Martín Servicio de Urología. Fundació Puigvert

Cartagena, 340-350 - 08025 Barcelona. Tel.: 934169700

E-mail autor: fsanchez@fundacio.puigvert.es

Información artículo: Original - Iniciativa Open Access

Trabajo recibido: marzo 2009

Trabajo aceptado: abril 2009 